\title{
Analysis of Research Trends Related to Child Counseling
}

\author{
Yoon-Jeong Cha ${ }^{1}$, Mi-Na Lee ${ }^{2}$ \\ ${ }^{1}$ The Doctor's Course, Department of Human Service Education, Kwangshin University, South Korea, \\ cowner@hanmail.net \\ ${ }^{2}$ Professor, Welfare Counseling Convergence Department, Kwangshin University, South Korea, \\ lmn4780@naver.com
}

Corresponding author: Mi-Na Lee

\begin{abstract}
The study analyzed research trends related to child counseling mainly based on doctoral dissertations in Korea. The study analyzed collected research data by year, media type, and address factors focusing on domestic doctoral dissertations. The research year surveyed was from 2000 to 2020 with a total of 709 publications. The research issues were examined according to the trends of child counseling-related degrees, year, media type, and residential address factors. First, in terms of research trends by year, it was the highest with 67 articles (9.4\%) in 2019. The results of research analysis by media type were 281 articles $(35.2 \%)$ on art media (using natural objects, cognitive-behavioral approach, solution-centered and reality therapy approach, etc.) The research trend of each home appeal factor related to child counseling was found on 279 articles $(33.7 \%)$ on emotional factors (ADHD, behavioral disorder, depressive lethargy, autism and autism spectrum, anxiety, etc.). This can be said to be a part that emphasizes the specificity and expertise of counseling for children in the developmental process. In addition, since verbal self-expression is in the process of development, one can state that counseling using more diverse media is emphasized. Lastly, as in the analysis of research trends by home appeal factor, it suggests that the area of child counseling should be more specialized in emotional factors (ADHD, behavioral disorder, depressive lethargy, autism and autism spectrum, anxiety, etc.).
\end{abstract}

Keywords: Child Development, Child Counseling Expertise, Trend Analysis, Media Type, Residential Appeal Factor

\section{Introduction}

Child counseling is counseling for children. A child counselor should fully reflect the knowledge and understanding of child development psychology, as the child is the primary caregiver. In the course of counseling, counselors have the most influence and are the most important players in forming counseling relationships[1]. Corey (2002) also said in a study that mentioned the role and qualifications of counselors that the most important tool in counseling is the counselor's role, and the most powerful technique is the counselor's ability. Therefore, the role of a counselor in the counseling process and performance is very important. In addition, counselors play an important role as partners who share a healing journey with the counselee in the counseling process, while counselee are also influenced by the counselor in the process. while counselors are also influenced by the counselor in the process. During the consultation process, the counselor solves problems related to the main appeal of the person and experiences repeated stress, emotional exhaustion, lethargy, and loss of motivation. In particular, counselors experience vicarious trauma or empathy fatigue as they indirectly suffer problems,

Received: February 19, 2021; 1st Review Result: April 02, 2021; 2nd Review Result: May 24, 2021

Accepted: June 30, 2021 
experiences, and trauma of the visitor and are constantly exposed to the visitor's trauma[2][3]. Counselors have been trained to care for the needs of others in the course of counseling and training, so they are used to ignoring the need for self-management and sacrificing themselves for the sake of the narrator[4]. In addition, counselors are negligent in managing stress and lack practice to take care of mental health[5] or needs[6][7]. Counselors experience psychological and emotional difficulties in performing their roles, and this experience reaches such a serious level that it causes the counselor's professional identity and individual psychological problems.

Child counselors show different characteristics in the content of stress due to differences in counseling targets from adult counselors. In child counseling, adults responsible for raising children play an important role in requesting counseling, participating in the counseling process, and solving the problem of the main appeal. Therefore, counselors include children and parents, or adults in charge of child care. Therefore, parental counseling should be carried out in child counseling, and the relationship between child counselors and parents or clients in the counseling process affects whether the consultation continues and the outcome of the consultation[8]. Children's counseling shall be essential to the counselor's ability to use various media, such as games, art, as counseling methods suitable for the developmental characteristics of children.

In addition, parent counseling requires professional knowledge and skills, such as parental counseling techniques, understanding adult development processes, and insight into outstanding problems of counselors who affect counseling. Therefore, child counselors need training in child counseling and parental counseling at the same time[9], i.e., the emotional stress experienced by counselors violates the counseling process and the rights and interests of counselors. Counselor ethics regulations refer to the minimum level of ethical commitments that should be kept while performing professional tasks as counselors, and are also necessary for the counselor's decision-making process. Therefore, the inability of counselors to take care of themselves means that they violate both the maintenance of their identity as counselors and the ethics of counseling. Child counselors who consult with children have different experiences, professional characteristics, and stress experiences from counselors for adults. The following are differences between adult counselors according to the occupational characteristics of child counselors: First, it is characterized by the lack of spontaneity of children in counseling. This is not because children themselves recognize the problem and visit the counseling center, but because their parents, who are their guardians, visit the counseling center. Due to involuntary characteristics, children may be reluctant to consult due to lack of motivation, easily frustrated, and resisted in the counseling process. These characteristics not only cause child counselors to experience negative emotions in the child at home[10], but also prevent them from establishing therapeutic partnerships[11]. Second, children are still developing and are characterized by being immature. Children are in the process of development and are immature in verbal communication and self-expression, making it difficult to express their desires in language during the consultation process[12].

Therefore, more nonverbal communication is required during the consultation process, so actual child counselors are more stressed because the inpatient is the child than the child's problem to be solved. In other words, child counselors need more specialized skills because they need to conduct counseling for children who have difficulties in communication. To lead to this change in children, counselors need not only professional knowledge and skills, but also understanding and experience in various cultures that can help them understand[13]. In the same vein, children have regressive characteristics, so child counselors not only reverse the counseling process but also have negative inner feelings such as anger, helplessness, and embarrassment more frequently than adult counselors. Third, consult their parents with their children and, if necessary, deal with the environment surrounding their children at the same time. Child counselors require a variety of approaches, including dealing with children's difficulties and consulting their parents and continuing to interact with their families, peers, teachers, etc.[14].

The goal of child counseling is to improve problem solving skills by improving children's self-esteem 
while alleviating and solving the difficulties that children are currently facing. The role of parents is very important in this process, and the overall goal of parental counseling is to understand children's difficulties, practice and maintain appropriate parenting attitudes, and have proper parenting effectiveness in this process. To this end, child counselors need knowledge and skills in understanding parental counseling techniques and adult development processes, so child counselors need training courses in parental counseling at the same time. In other words, child counselors are forced to experience the burden and stress of counseling in this process, as they must be accompanied by constant parental counseling and education[15].

\section{Theoretical Background}

\subsection{The Developmental Characteristics of Childhood}

Since birth, humans interact with various others as members of their own society, acquire and internalize the values, norms, and patterns of their groups, form their own selves and grow into members of society. Childhood experiences a gradual expansion of human relationships away from home-oriented life and social relationships with other people and peer groups. In the process, you will develop and develop your own unique self through the learning process of developing and modifying behavior according to the behavior patterns of your group members. Childhood, in particular, increases selfrespect and verification of reality through interaction with peer groups, and feels a sense of belonging. They reserve selfishness and improve social cooperation attitude and social skills. Among the stages of human development, childhood is the most important period for social and emotional development, and the basic social and emotional development formed during this period is very important for future interpersonal building and development as a competent social member. However, it is easy to experience frustration and developmental crisis in the face of difficulties in building self-respect if one is not supported by a friend or peer group and is ostracized[16].

Child counseling is a branch of mental health services to help children develop healthy growth. In other words, child counseling is a specialized area that helps children directly and indirectly to enhance the functioning of children and family members to maintain the family framework and enhance their function within it[17]. A child counselor shall also be a person engaged in the field of counseling and psychotherapy, and may discuss expertise in a similar context. However, just as expertise is based on the development of "area specific" development, so is child counseling in the counseling area. Therefore, child counselors' expertise should be described by including the specificity of child counseling in addition to the discussion of expertise in general adult counseling.

The specificity of child counseling includes the characteristics of children subject to child counseling and the clinical environment of child counseling. We explain the specificity of children in that they are not well-developed in language, are in the development process, child development has integrated characteristics, and the handling of children within a system should consider various environments[18]. Child counseling requires special ability of counselors to understand the characteristics of children and the developmental and environmental context, so child counselors should be familiar with it.

It may be explained that child counseling is distinct from adult counseling as follows:

First, since children are growing up, child counseling should be approached from a different perspective than counseling for adults. Children's problems should be considered in the context of growth and development, and child counseling and psychotherapy work should also consider these characteristics. Second, children lack language skills than adults, so the approach of child counseling is used by different media than adult counseling, which is based on language conversation. Access to play therapy, art therapy, music therapy, etc. is an effective counseling approach that takes into account the characteristics of such child counseling. Third, children are passive unlike adults who voluntarily visit 
counseling, which is indicated by differences in counseling motivation. Fourth, children's problems often require multi-dimensional consideration of their relationships or environmental contexts, and parental changes or assistance in counseling affect the outcome of counseling. Fifth, special counselors' ability to understand the mental world of children is required. Sixth, children have the potential to choose the future, which is based on the fact that children are still in the process of growth, while adult counseling is a counseling for solving problems, child counseling adds intervention for growth. In other words, child counseling can vary in the attitude, strategy, and approach of the counselor depending on the developmental and behavioral characteristics of the child[8]. In particular, unlike adult counseling, child counseling requires more nonverbal communication methods, including language, and has the characteristics that supporting relationships with the main caregiver or parent are important. In addition, child play therapy[19], which accounts for the highest percentage of child counseling approaches in Korea, uses play as a therapeutic tool as well as the counselor himself, so factors such as healer playability are characteristic of play therapy professionals. In the domestic child counseling scene, parental counseling is held regularly and many counseling strategies[20] are used to promote changes in children through parents, so the factors of parental counseling should also be considered important. Child counselors have said that it is important for child counselors to work with parents because they can determine the role of parents and improve their role skills to promote children's growth.

\subsection{A Child's Psychopathology}

The process of child counseling refers to a counseling process that is varied according to the nature and situation of the child's problem, the counselor's theoretical background, and the counselor's and client's desires and values. In simple cases, it can be completed in one or two interviews, but for psychological problems, it may take 5, 6 to 20 or even more years depending on the severity. The child counseling process is explained by each counselor according to their own theoretical basis, and most of the counseling process can be divided into three stages: early, middle, and late.

The initial stage aims to establish a therapeutic environment, establish a therapeutic relationship, and agree on counseling goals. Counseling skills mainly used in the early stages are acceptance, reflection, empathy, silence, clarification, questions, use of non-verbal clues, and role play. In the early stage, when the therapeutic environment and the goal of counseling are formed, the process moves to the mid-term stage. The main goals of this stage are specific and practical things for full-fledged problem solving, such as profound exploration and analysis, understanding of the child's world and lifestyle, and assistance in achieving counseling goals. In the mid-term, suggestions, information, interpretation, unfinished thoughts, questions, temporary analysis and temporary hypotheses, and self-exposure are used as counseling skills.

Moving on to the final stage of counseling, which is the stage of achieving the goal through the child's actions. At this stage, children learn and develop to behave in a constructive way toward themselves and others. The main goals at the end of the stage are a summary and evaluation of the results and achievements that have been worked to reach the counseling goals, and a successful conclusion. The decision to terminate the consultation is a very important issue at this stage. Clear standards need to be set in order to establish a standard for whether to continue or close the consultation. At this stage, it is important for counselors to recognize that the child can get help again whenever he or she wants. In the late stages, problem-solving, decision-making, confrontation, immediateness, and encouragement are used as key counseling skills. Above all, encouragement requires a lot of patience from the counselor, and it is very important to wait.

A child counselor should acquire knowledge of several mental disorders of childhood that appear in childhood development and should steadily strengthen his or her expertise. DSM-IV separately 
identified childhood disorders as ten disorders of mental retardation, overall developmental disorder, attention deficit and destructive behavior disorders, learning disorders, communication disorders, tic disorders, excretion disorders, feeding and diet disorders, and motor skills. However, DSM-5 as amended in 2013 does not diagnose childhood and adolescent mental disorders separately from other disorders, adding a new category of disorders as neurodevelopmental disorders and other mental disorders[21].

Attention Deficit Hyperactivity Disorder (ADHD) was classified as a neurological disorder with a later revision to DSM-5, with a sociocultural problem and pathology at the same time, which tends to focus on sociological and destructive behavioral disorders. ADHD sees carelessness, hyperactivity and impulsivity as key symptoms of disability, and DSM-5 distinguishes ADHD from three subtypes: attention deficit type, hyperactivity and impulse type, and mixed type[22]. ADHD children are particularly poor at adapting to school life, showing interpersonal or behavioral problems, and $30 \%$ to $50 \%$ of ADHD children aged 7-10 show demeanor and antisocial behavior. ADHD children tend to be unable to sit quietly, pay attention to teachers or follow instructions, suppress impulsive behavior, cooperate with other students, experience difficulties in collective action, and perform routine activities responsibly. The prevalence of ADHD is about $5 \%$ for children, with male children having six to nine times higher prevalence than female children[23].

Dignity disorder refers to a case in which a violent or irresponsible act, such as a continuous repetition of acts that violate other people's basic rights or violate social norms appropriate for their age, causes other people's pain repeatedly. It usually appears in a variety of aggressive behaviors, a fairly common disorder in childhood and adolescence, and is common in male children. Dignity disorders in childhood affect a variety of factors, such as poor family conditions including parental discord, domestic violence, child abuse, and coercive or neglectful parenting of parents[24].

Counseling-related institutions in Korea include the Korea Counseling Association, the Korea Counseling Psychology Association, the Korea Christian Psychological Counseling Association, the Korea Exchange Analysis Counseling Association, the Korea Reading Therapy Counseling Association, and the Korea Integrated Art Therapy Counseling Association. Among them, the Korea Counseling Association's Code of Ethics (2002) emphasized that self-care of counselors is ethically essential, stating that counselors should not start counseling when they cannot properly help counselors due to their limitations or personal problems.

The American Psychological Association (APA, 2002) also emphasizes self-care in terms of ethical responsibility and readiness, suggesting that 'psychologists should refrain from counseling when they perceive or judge that their personal problems may interfere with counseling in legal ways associated with counseling'. In the Code of Ethics (2005) of the Korean Society for Play Therapy, "Play therapists sometimes find that they cannot provide appropriate help to children with certain internal affairs." In such cases, the play therapist should inform the appropriate requesting agency. As it is specified, child counselors are required to protect children and child counselors by making appropriate requests and closing.

Through this, we would like to propose useful information to conduct research on child counseling by analyzing and investigating the research trends on child counseling, focusing on domestic academic papers that have been studied.

This study intended to analyze the research trends of children counseling research, focusing on the doctoral dissertations published in Korea from 2000 to 2020, and the research problems are as follow.

First, what is the distribution of annual doctoral dissertations related to child counseling?

Second, what are some characteristics of the field of research on media usage related to child counseling?

Third, what are the characteristics of research by major appeal factors related to child counseling? 


\section{Research Method}

\subsection{Analysis Target}

This study analyzed the domestic doctorate papers on child counseling in electronic libraries, RISS4U, KISS, and National Library of Korea Research Foundation and National Assembly Library. Keywords used in the collection process were children's counseling, child psychological counseling, child play therapy, children's art therapy, children's reading therapy, children's therapy, child development therapy, and children's counseling programs. A total of 709 doctoral dissertations were analyzed.

\subsection{Data Processing}

The data from this study were gathered from 709 doctoral dissertations related to child counseling from 2000 to 2020 . In order to identify general trends, data were analyzed by degree type, year, media type, and main appeal factor in a statistical manner.

In addition, research papers related to child counseling are processed as data using an Excel program to analyze them in accordance with the analysis criteria table for each element. At this time, the analysis results were analyzed frequently by mistake and percentage, and the percentage of all analysis tables were rounded to the first place.

\section{Analysis Result}

\subsection{Research Trends by Year of Child Counseling}

Annual research trends are analyzed for children's counseling research by degree type. When looking at the dissertations and frequency of child counseling research, the annual breakdown and percentage of doctoral dissertations from 2000 to 2020 is presented as shown in [Table 1].

[Table1] Degree Thesis Children's Counseling Research Year-by-year Classification

\begin{tabular}{ccccccccccccc}
\hline Year & 2000 & 2001 & 2002 & 2003 & 2004 & 2005 & 2006 & 2007 & 2008 & 2009 & 2010 & Subtotal \\
\hline $\begin{array}{c}\text { Disse } \\
\text { rtatio } \\
\mathrm{n}\end{array}$ & 4 & 9 & 6 & 18 & 10 & 21 & 16 & 23 & 22 & 25 & 31 & 185 \\
\hline $\begin{array}{c}\%) \\
(6\end{array}$ & 1.3 & .8 & 2.5 & 1.4 & 3.0 & 2.3 & 3.2 & 3.1 & 3.5 & 4.4 & 26.1 \\
\hline \hline \begin{tabular}{c} 
Year \\
\hline $\begin{array}{c}\text { Disse } \\
\text { ratio } \\
\mathrm{n}\end{array}$
\end{tabular} & - & 2011 & 2012 & 2013 & 2014 & 2015 & 2016 & 2017 & 2018 & 2019 & 2020 & - \\
\hline $\begin{array}{c}\% \\
(\%)\end{array}$ & - & 45 & 37 & 52 & 44 & 60 & 57 & 57 & 49 & 67 & 66 & 524 \\
\hline $\begin{array}{c}\text { Subto } \\
\text { tal }\end{array}$ & 4 & 44 & 43 & 70 & 54 & 81 & 73 & 80 & 71 & 92 & 97 & 709 \\
\hline
\end{tabular}

The analytical data collected as shown in [Table 1] collected a degree research paper on child counseling published from 2000 to 2020. There were a total of 709 doctoral dissertations, the highest with $67(9.4 \%)$ in 2019, $66(9.3 \%)$ in 2020, 60 (8.5\%) in 2015, $57(8.0 \%)$ in 2016 and $57(8.0 \%)$ in 2017 , and $4(0.6 \%)$ in 2000 , which is the lowest. 


\subsection{Research Trends by Media Type Related to Child Counseling}

The research trends of each type of media are analyzed for the degree thesis related to child counseling. Trends by type of media related to child counseling may be presented as shown in [Table 2].

[Table 2] Comparison of Research Trends by Types of Child Counseling Media

\begin{tabular}{|c|c|c|c|}
\hline \multirow{2}{*}{\multicolumn{2}{|c|}{ Sortation }} & Thesis & \multirow{2}{*}{$\begin{array}{l}\text { Dissertation } \\
\text { rate }(\%)\end{array}$} \\
\hline & & Doctor & \\
\hline Play media & Parent-child, child-centered, play in motion, play games, etc. & 221 & 27.7 \\
\hline Art medium & $\begin{array}{l}\text { Utilization of natural objects, cognitive behavioral approaches, } \\
\text { resolution centers, approaches to real-world therapy, etc. }\end{array}$ & 281 & 35.2 \\
\hline Sand media & Personal counseling, school, group, in-house counseling, etc. & 77 & 9.6 \\
\hline Reading media & Interactive, picture books, integrated methods, etc. & 74 & 9.3 \\
\hline $\begin{array}{l}\text { Language } \\
\text { medium }\end{array}$ & Language therapy, language rehabilitation, verbal violence, etc. & 97 & 12.1 \\
\hline $\begin{array}{l}\text { Expression art } \\
\text { medium }\end{array}$ & Role play, psychological play, motion expression, etc. & 49 & 6.1 \\
\hline & Sum & $799 *$ & 100 \\
\hline
\end{tabular}

* Identified as a duplicate study

As shown in [Table 2], research and analysis by type of media related to children's counseling showed that art media (natural water treatment, cognitive behavioral approach, resolution-centered and practical therapy approach) is the highest with 281 (35.2\%) followed by play media with $221(27.7 \%)$ and language medium with 97 (12.1\%).

\subsection{Comparison of Children's Counseling Related Factors in the Main Appeal Center}

The trend of child counseling-related appeals is analyzed by factors related to children's counseling. Research trends by type shown by main appeal factors are analyzed by language factors, attachment factors, school-related factors, maladaptive and aggressive factors, social norm factors, emotional factors, and physical factors. The research trends by factors of child counseling centers are as shown in [Table 3].

[Table 3] Research Trends by Factors of Child Counseling-Related to Child Counseling

\begin{tabular}{cccc}
\hline Sortation & \multicolumn{2}{c}{ Thesis } & Dissertation rate(\%) \\
\cline { 2 - 3 } $\begin{array}{c}\text { Language therapy, language rehabilitation, } \\
\text { language development delay, etc. } \\
\text { Verbal violence, language prevention } \\
\text { counseling, etc. }\end{array}$ & 168 & 153 & 18.6 \\
\hline $\begin{array}{c}\text { Sexual problems (sexual violence, sexual harassment, etc.) } \\
\text { Attachment related (unstable attachment, peer attachment, etc.) }\end{array}$ & 6 & 20.4 & 1.8 \\
\hline Peer relationship (inadaptation, communication, etc.) & 62 & 7.7 \\
\hline $\begin{array}{c}\text { Factors related to school life } \\
\text { (Vulnerable, Elementary) }\end{array}$ & 19 & 2.3 \\
\hline Victim student & 34 & 22 & 2.7 \\
\hline
\end{tabular}




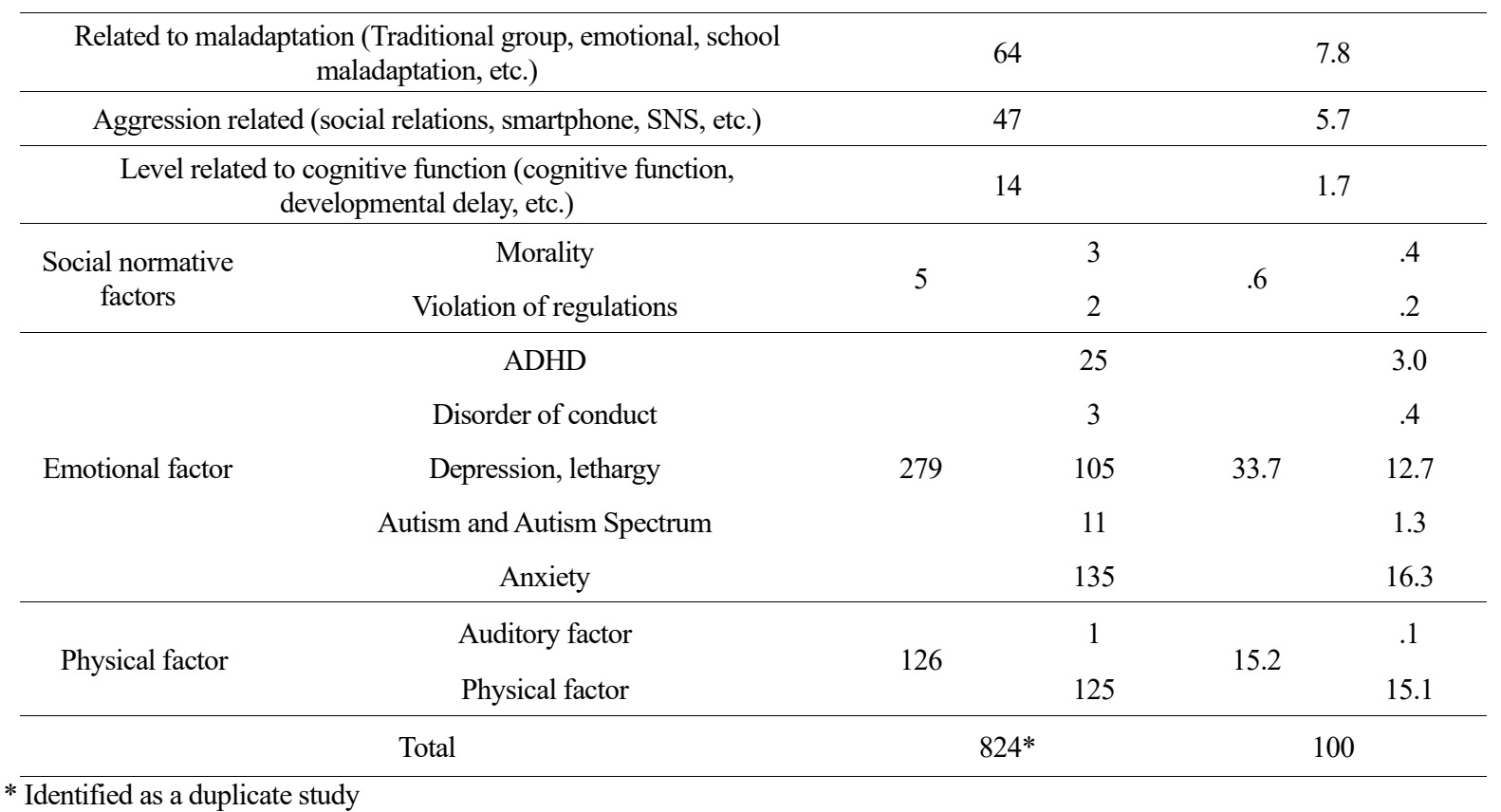

According to [Table 3] of the main appeal factors related to child counseling were $279(33.7 \%)$ emotional factors (ADHD, demeanor, depressive lethargy, autism and autism spectrum, and anxiety), 168 (15.4\%) language factors (language development, language therapy, language rehabilitation, and language violence) are found, $34(4.2 \%)$ factors related to school life, $19(2.3 \%)$ peer-related factors (e.g., inadaptation and communication), 14 (1.7\%) cognitive factors (e.g., cognitive function and developmental retardation), $6(0.7 \%)$ sex-related factors (e.g., sexual violence and sexual harassment), and $5(6 \%)$ social norm factors (e.g., moral and rule violation).

\section{Conclusion}

Children have a lot of difficulties in that their language skills are still less developed, their motivation for psychological treatment is low, and their understanding is not well expressed. A lot of researches have been conducted to overcome this problem, and this field has been created according to the achievements. It can be largely divided into play therapy and behavior modification. In play therapy, a therapeutic human relationship is formed, desires and emotions are liberated so that children can easily interpret them and lead insights through play. Play is a child's life, words, and a place where desires and emotions are released. In play therapy, environment creation is also important, and in particular, the change of mother's attitude toward children is emphasized.

Behavior modification is based on the theory of learning to reinforce desirable behaviors or eliminate undesirable behaviors to lead to changes in behavior. Child psychological treatment is mainly applied to neurotic habits, emotional disorders, shedding, neurosis, and social disorders. The child psychotherapist makes a comprehensive diagnosis through scientific measurement tools or various psychological tests for children with various disabilities and problem behaviors, and provides psychological counseling and treatment activities according to each situation and environment. Heals disabilities and problems. In addition to play therapy and behavior modification, speech therapy and art therapy are often performed to identify and treat children's problems and psychological conditions.

Child counseling is a branch of mental health services to help children develop healthy growth. In other words, child counseling is a specialized field that helps children directly and indirectly to enhance the function of children and family members to maintain the family framework and strengthen their functions within the framework. In addition, the specificity of child counseling may include the 
characteristics of children subject to child counseling and the clinical environment of child counseling, which requires more professional counseling because children are not fully linguistic.

Specificity and expertise should be emphasized in that children are in the development process, child development has integrated characteristics, and the handling of children in the system should take into account various environments.

In the annual research trend for doctoral dissertations on child counseling, 67 (9.4\%) published in 2019 are collected and analyzed from 2000 to 2020. The research and analysis results for each type of media are 281 artworks (35.2\%) (e.g., natural water utilization, cognitive behavioral approach, solutioncentered, and practical therapy approach, etc). Research trends by factors related to child counseling are 279 emotional (33.7\%) factors (ADHD, demeanor, depressive lethargy, autism and autism spectrum, and anxiety).

This can be said to emphasize the specificity and expertise of counseling for children in the development process. In addition, since verbal self-expression is in the development process, counseling using more diverse media is emphasized. Finally, as in the analysis of research trends by major appeal factors, sentiment factors (e.g., ADHD, demeanor, depressive lethargy, autism and autism spectrum, and anxiety) are the result of suggesting that the area of child counseling should be more specialized.

Based on the limitations of this study, the direction of subsequent studies can be presented. A study shall be conducted to collect and analyze data from existing research on topics related to child counseling. Research on relevant concepts needs to be included in order to establish a clearer concept. Thus, investigative research should be combined with theoretical approaches. Practical application studies would be reasonable in the direction of subsequent studies as these trend research approaches should lead to solving child counseling areas.

\section{References}

[1] T. M. Skovholt, The resilient practitioner: Burnout prevention and self-care strategies for counselors, therapists, teachers, and health professionals, (1st edition), Allyn \& Bacon, (2000)

[2] L. A. Pearlman, K.W. Saakvitne, Treating therapists with vicarious traumatization and secondary traumatic stress disorders, (1st edition), Compassion fatigue, (1995), pp150-177.

[3] B. H. Stamm, Secondary traumatic stress: Self-care issues for clinicians, researchers, and educators, The Sidran Press, (1995)

[4] T. M. O'Halloran, J. M. Linton, Stress on the job: Self-care resources for counselors, Journal of Mental Health Counseling, (2000), Vol.22, No.4, pp.354-364.

[5] C. Maslach, Burnout The Cost of Caring, Englewood Cliffs, NJ Prentice-Hall, (1982)

[6] J. Baker, Essential Speaking Skills: A Handbook for English Language Teachers, Diane Publishing Company, (2005)

[7] C. R. Figley, (Ed.). Treating compassion fatigue, (1st edition), Rutledge, (2002)

[8] K. W. Kim, A Preliminary Survey for the Effective Parent Consultation in Play Therapy, Korean Journal of Play Therapy, (2009), Vol.12, No.1, pp.47-63, UCI : G704-SER000000779.2009.12.1.007

[9] K. M. Hong, K. W. Kim, A research study on the concept of the Counselor`s self-care and related variables, Korean Journal of Play Therapy, (2012), Vol.15, No.2, pp.153-177, UCI : G704-SER000000779.2012.15.2.004

[10] G. O. Kim, K. W. Kim, A Study on Development Scale of Child Client's Resistance perceived by Play Therapist, Korean Journal of Play Therapy, (2010), Vol.13, No.2, pp.113-138, UCI : G704-SER000000779.2010.13.2.002

[11] M. S. Choi, K. W. Kim, The Therapeutic Relations and Outcomes of Playtherapy, Korean Journal of Play Therapy, (2007), Vol.10, No.1, pp.47-62, UCI : G704-SER000000779.2007.10.1.003 
[12] H. J. Han, K. W. Kim, Empathy of Playtherapists and Emotional Bond Felt by Client Children with Therapists, Korean Journal of Play Therapy, (2004), Vol.7, No.1. pp.77-89.

[13] E. M. Joung, An Analytic Study on Parent Education Program Utilizing the MBTI Personality Test, Journal of Parent Education, (2016), Vol.13, No.1, pp.79-92.

[14] H. S. Park, Yeongae Lee, The Effect of Five Personality Factors of Play therapist on the Burnout, Korean Journal of Play Therapy, (2009), Vol.12, No.2, pp.35-47, UCI : G704-SER000000779.2009.12.2.007

[15] J. H. Choi, H. A. Kang, The effect of job stress and personal resources of child counselors on burnout and job enthusiasm, Journal of Korea Child Care and Education, (2012), Vol.8, No.5, pp.231-252, UCI : G704SER000010376.2012.8.5.001

[16] E. S. Cho, The effect of learning environment variables on elementary school students' science inquiry ability, Korea National University of Education, Master's thesis, (2000)

[17] M. S. Yoo, Y. S. lee, E. S. lee, J. J. Choe, J. H. Choei, The therapeutic power of play: 20 key mechanisms for change, Sigma Press, (2015), ISBN9788968664113

[18] S. H. Kim, Modern Child Counseling, Hakjisa, (2004)

[19] Jae-Ryung Yoo, Gwang-Woong Kim, The Education/training Background as a Variable Related Ethical Behaviors of Child Counselors, Korean Journal of Child Studies, (2008), Vol.29, No.1, pp133-153, UCI : G704000080.2008.29.1.010

[20] M. S. Yoo, M. S. Choi, Y. K. Joung, Qualitative study about Supervision Experience of Experienced play-therapist, Korean Society for Play Therapy, (2009), Vol.12, No.4, pp19-42, UCI : G704-SER000000779.2009.12.4.003

[22] H. G. Shin, J. S. Kim, Attention deficit and hyperactivity disorder, Hakjisa, (2000)

[23] T. H. Kim, How to respond to an aging society, Samhwa Publishing House, (2003)

[24] Lauren B. Alloy, John H. Riskind, Margaret J. Manos, Ideal Psychology: The Present Perspective, ParkHaksa, (2010) 